\title{
Evolution and classification of the mountain Caddis-flies
}

\author{
(University of Illinois Press, Urbana, 1956)
}

H. . H. ROSS

La parution de cet ouvrage marquera certainement une date importante dans l'histoire de la trichoptérologie, non seulement par la somme considérable de connaissances qu'il apporte et par l'étendue des problémes qu'il traite, mais surtout par l'esprit nouveau qu'il introduit dans ce champ d'investigation. Jusqu'ici, la plupart des travaux sur les Trichoptères ont été avant tout descriptifs et le petit nombre de ceux qui ont été consacrés à la phylogénie et à la zoogéographie ont traité les questions d'un point de vue surtout statique.

Or, dans son ouvrage, Ross envisage les problèmes de façon dynamique et s'applique à retracer l'évolution des groupes qu'il étudie, en fait à en reconstituer l'histoire, sous la forme de séquences phylétiques retracées étape par étape: L'ouvrage produit une excellente impression par sa présentation brillante et par sa haute tenue. Le texte est écrit dans un style clair, précis et personnel; il est accompagné d'un grand nombre de figures qui illustrent les développement reconstitués et de nombreux arbres phylétiques, souvent fort intelligemment présentés, qui résument la différenciation des lignées et leurs dispertions géographiques à des époques déterminées.

En parcourant les pages de Ross nous trouvons tout d'abord expliqués un certain nombre de concepts, dont la clarification est indispensable aux études phylétiques et zoogéographiques, puis nous assistons à une véritable reconstitution de l'histoire de l'ordre. Des essais très intéressants traitent de 1'origine, des moeurs et de l'habitat des Trichoptères primordiaux. Les caractères ancestraux des trois stades sont reconstitués et Ross explique, sur la base de caractères fondamentaux, quelles on été les premières divisions de l'ordre et les principales directions d'évolution qu'ont suivi les điverses lignées. Ces sujets n'avaient jamais été effleurés auparavant. L'auteur donne également un arbre phylétique de l'ordre, le premier qui soit apparu dans la littérature.

La partie systématique est consacrée à l'étude des trois familles les plus primitives, les Philopotamides, Rhyacophilides et Glossosomatides. C'est-là le premier travail d'ensemble sur ces trois groupes dont, jusqu'ici, nous n'avions guère idée de la classification. Non seulement Ross donne une diagnose de chacun des genres et la liste des espèces qu'il contient, mais il reconstitue la phylogénie de chaque genre et de chaque famille à l'aide des caractères morphologiques de la nervulation et des génitalia. Souvent, l'auteur fait montre d'originalité en basant ses considérations sur des caractères dont l'étude avait été négligée avant lui.

Dans la troisième partie enfin, à l'aide d'une riche documentation zoogéographique et géologique et en intégrant les données zoogéographiques et phylétiques, Ross arrive à dater l'apparition de beaucoup des lignées et leurs dispersions entre les continents. L'auteur se trouve donc en position d'aborder ensuite problèmes plus généraux : les carac- 
tères des périodes de dispersion, les conditions requises pour ces dernières, les ponts de Behring, les effets des glaciations, etc., et il traite ces sujets avec maitrise.

Ces connaissances sont dégagées de l'étude d'insectes habitant les montagnes et vivant dans des biotopes froids. Or, il se révèle que si de nombreux travaux ont été consacrés à la zoogéographie des insectes des régions chaudes, l'ouvrage de Ross est le premier qui ait tenté avec succès l'analyse des données fournies par les "cold adaptated biota" et les résultats en sont passionnants. En terminant, Ross émet l'espoir que son ouvrage stimulera l'intérêt des chercheurs pour les problèmes qu'il a traités. C'est non seulement ce que je souhaite avec lui, mais j'espère que beaucoup d'auteurs adopteront sa façon d'envisager les problèmes sous l'angle dynamique et historique.

Parmis les réserves, je dirai que sous la clareté et la concision du style apparait un raisonnement un peu superficiel, que dans ce moule brillant a été coulée une pensée un peu maigre et, qu'à l'étude, les conclusions de Ross inspirent souvent un certain scepticisme qu'accompagne parfois la déception.

Dans ses études taxonomiques, Ross utilise surtout, comme on le fait pour composer des tables dichotomiques, des caractères de détail, immédiatement visibles, qui lui ont parfois masqué des structures fondamentales, moins faciles à déceler, mais de plus grande importance phylétique. En particulier, il n'a apparamment pas cherché à établir d'homologies entre les appendices des diverses lignées. Il semblerait que 1'auteur ait manqué d'esprit synthétique et ait ell une vision trop partielle, trop superficielle et trop simple des. choses. Précisément, devant le mot "simple), qui revient constamment dans le chapitre consacré aux Rhyacophila, on en vient à se demander si la recherche de la simplicité ne serait pas, en fait, une exigence de l'esprit de l'auteur. Si ce dernier trouve les caractères primitifs qu'il cherche dans les formes simples, ne serait-ce pas parce que la simplicité exerce une sorte d'attraction sur son esprit et répond à une façon de penser qui lui est propre? Un tel doute s'insinue aussi dans le lecteur lorsqu'il étudie les arbres phylétiques. Si ces derniers ont souvent un axe linéaire, peut-être est-ce parce que la pensée qui les a construit tend à suivre une voie linéaire, à tenir compte d'un petit nombre de caractères seulement, à les considérer un à un et à les interprêter d'une certaine façon, alors qu'une autre, qui semblerait tout aussi acceptable ou même plus logique, a été négligée, sans que l'on sache les raisons de ce choix.

$\mathrm{Si}$, comme je le répète plus loin, beaucoup de solutions données comme des faits établis, inspirent au lecteur un sentiment de malaise et d'artificialité, ou de quelque chose qui "sonne faux", c'est qu'elles dépendent trop visiblement de la façon qui a été employée pour les déduire et qu'il est trop évident que l'auteur a projeté sa façon de penser dans ses constructions. A l'étude, les hypothèses zoogéographigues avancées par Ross apparaissent toujours logiques, mais parfois trop vagues, car elles ne serrent pas les faits d'assez près. Les reconstitutions phylétiques et les inférences de Ross sont vraiment trop faciles pour donner l'impression d'être ce qu'elles devraient, des synthèses délicates, résultats d'une patiente distillation et d'une lente maturation des idées dans l'esprit et le subconscient. 


\section{Moeurs des Trichoptères primordiaux}

Reconstituer ce qu'ont pu être les moeurs des premiers Trichoptères est certainement une tentative fort intéressante. Voici un résumé des résultats obtenus par Ross à ce sujet. L'auteur constate que les larves de tous les Annulipalpes fabriquent des abris fixes et ont des pattes anales allongées et capables de mouvements indépendants. Chez les Intégripalpes, les larves de la famille primitive vivent sans protection et ont des pattes anales semblables à celles des Annulipalpes. Celles des familles plus spécialisées construisent des étuis que l'on trouve de plus en plus perfectionnés quand on monte dans l'échelle systématique et, parallèlement, les pattes anales se spécialisent aussi bien dans leur forme que dans leur fonctions. Ross en conclus que: I) Les Trichoptères primordiaux avaient des pattes anales allongées et libres comme celles des Annulipalpes. 2) Et donc fabriquaient des retraites fixes comme celles des Annulipalpes également.

Le premier point est parfaitement correct. Trouvant des pattes anales identiques à la base des deux lignées, nous pouvons considérer ce fait comme une preuve que les Trichoptères ancestraux avaient des pattes anales allongées et libres. Mais, le second point est probablement incxact et non seulement le raisonnement de Ross ne convainct pas, mais il ne prouve rien. Car, si les premiers Trichoptères vivaient sans protection sur ou sous les pierres, il n'est nullement certain que les pattes anales des Annulipalpes auraient dûu alors se modifier en corrélation avec la vie en abri.

I a plupart des auteurs considèrent comme fixes les larves vivant en abris fixes et comme mobiles les larves vivant dans des étuis transportables. Or, du point de vue des relations larve-édifice de protection et, plus encore, du point de vue des pattes anales, c'est le contraire qui est correct. Les abris des Annulipalpes sont beaucoup plus grands que les larves qui possèdent une grande aisance de circulation à leur intérieur et en sortent facilement. Les étuis des Intégripalpes sont d'étroits fourreaux d'où la larve ne sort pas ordinairement, mais où il importe qu'elle soit solidement ancrée afin de pouvoir se livrer sans déplacement aux mouvements respiratoires ondulés. Ce sont donc les Annulipalpes qui ont des larves mobiles et les Intégripalpes qui ont des larves fixes, par rapport au substrat des pattes anales.

Nous trouvons donc chez les Trichoptères un type assez uniforme de pattes anales primitives, permettant une vie mobile sur un substrat naturel (Rhyacophilides) ou aménagé (les abris des Annulipalpes) et plusieurs types de pattes ancreuses, permettant une vie fixée à l'intéricur de constructions artificielles (le's fourreaux des Intégripalpes). Il me parait donc clair que ia présence de pattes anales primitives est en relation avec une existence mobile et avec ce caractère seulement. Ou alors, les Annulipalpes auraient, comme les Intégripalpes, développé plusicurs sortes de pattes anales, adaptées aux différents types d'abris qu'elles construisent et qui sont plus variés que les étuis des Integripalpes: sacs larges et souples des Philopotamides, tubes longs et étroits des Psychomyides ou nasses rigides des Hydropsychides, etc. Le fait que les Annulipalpes aient des pattes anales semblables à celles des ancêtres de l'ordre ne prouve donc pas que ces ancêtres 
étaient des bâtisseurs d'abris, mais simplement qu'ils avaient des larves douées d'une grande aisance de circulation.

La troisième conclusion de Ross, amenée par la deuxiène est qu'une lignée primitive de "retreat-makers" a perdu la faculté de fabriquer des abris, a vécu libre sous les pierres comme les Rhyacophilides actuels (première spécialisation) et ensuite seulement a donné naissance à une lignée où s'est développée la faculté de construire cles étuis (deuxième spécialisation). Ces derniers, et ceux-là seulement, seraient une spécialisation de l'abri nymphal.

Mes deux remarques sont: I) $\mathrm{Si}$ mes objections expliquées ciavant étaient correctes, plus rien ne justifierait cette troisième conclusion. 2) Les Annulipalpes forment une unité systématique beaucoup plus homogène que les Intégripalpes et, à la base de cette lignéelà, les génitalia des adultes sont déjà nettement plus spécialisés que chez beaucoup de familles d'Intégripalpes.

Mon opinion serait plutôt que les larves des Trichoptères primordiaux étaient libres, étaient pourvues de pattes anales primitives et ne construisaient rien d'autre que des abris nymphaux, comme celles des Rhyacophilides actuels. En cela, elles avaient des moeurs assez semblables à celles des larves de Mégaloptères, mais avec cette différence, qui est une spécialisation, que le stade nymphal étant devenu aquatique chez les Trichoptères primordiaux, leur abri nymplial était une modification de la niche que les larves de Mégaloptères s'aménagent hors de l'eau. Les Trichoptères primordiaux auraient donné naissance à deux lignées, les Annulipalpes et les Intégripalpes, qui auraient développé parallèlement la faculté de construire des abris et des étuis. Ces deux types de protection seraient tous deux des développements secondaires et des prolongements, dans le temps et dans l'espace, de l'abri nymphal. J'irai même plus loin: j'inclinerai à penser que les Intégripalpes sont polyphylétiques et que la faculté de construire des étuis 's'est développée parallèlement chez les Glossosomatides et les Hydroptilides d'une part et chez le reste de Intégripalpes d'autre part, car ces derniers ne semblent pas issus de l'ancêtre des Hydroptilides, qui était déjà trop spécialisé, mais se sont développés à partir d'un ancêtre commun. Mais, la justification de ce dernier point nous entrâ̂nerait hors du cadre de cette critique.

\section{Habitat des Trichoptères primordiaux}

La (Chart 2) représente un arbre phylétique de l'ordre, qui montre que dans toutes les lignées les familles primitives vivent en eau froide alors que celles qui sont plus spécialisées ont émigré en eau chaude. La régularité de ce fait est frappante.

Mes commentaires seront, tout d'abord, que les arguments avancés par Ross à l'appui de cette hypothèse sont convainquants et qu'il apparait certain que, jusque au Crétacé au moins, tous les 'Trichoptères vivaient en eau froide et agitée où sont restés les groupes primitifs at que les lignées plus spécialisées ont assez tardivement colonisé de nouveaux biotopes aquatiques plus tempérés et moins oxygénés.

Mais; considérant la "Chart 2", je ferai deux réserves. I) Les exceptions et les erreurs y sont trop nombreuses. Ainsi, les Hydrobiosi- 
nes, supposées "chaudes", habitent, sauf $A$ topsyche et quelques $A p$ silochorema, ides régions froides et tempérées, où les eaux chaudes n'existent pas. En Inde, pays qui comprend une grande partie de l'aréal de Stenopsyche, ce groupe est "chaud" ou (frais) et ne s'élève pas en altitude au-dessus de $4.000 \mathrm{ft}$. La grande majorité des Dinarthrum sont "froids", etc. 2) I es rectangles terminaux de l'arbre phylétique représentent des unités systématiques d'importance très inégale. Comme il n'est pas de groupes figurés dans l'arbre qui ne connaissent pas d'exceptions, il est facile de détacher des rameaux familiaux, aux endroits où cela est nécessaire, le nombre voulu de genres "chauds" nécessaires à la constitution de la régularité mentionnée plus haut. S'il est un fait que les 'Trichoptères sont apparus en eau froide et se sont ensuite adaptés aux eaux qui le sont moins, ceci est moins régulier, moins constant et sans doute plus tardif que 1'a affirmé Ross.

Mais il est d'autres observations qui confirment 1'hypothèse de cet auteur, quoiqu'elles donnent des résultats également peu réguliers et assez flous.

Bcaucoup 'de groupes ont colonisés les eaux calmes et stagnantes des lacs, étangs et marais et cela indépendamment de la température de ces milieux. Ia plupart des Limnophilines, Phryganéides, beaucoup de Leptocérides, certains Hydroptilides, Psychomyines, Séricostomatines et Lépidostomatides sont des limnicoles froids. De très nombreux Leptocérides, Ecnomines, Hyalopsychines. Psychomyines, Macronematines et passablement d'Hyidropsychines et Hydroptilides sont des limnicoles chauds. Les très petits cours d'eau et les terrains détrempés et ruissclants sont habités par des Limnophilines, Beraeines, Hydroptilides, Ptilocolepines, dans les régions tempérées et par des Rhyacophilines, Philopotamides, Agapetines, Psychomyines, Leptocérides, Lépidostomatides et Hélicopsychines dans les régions subtropicales. Les milieux hydropétriques ont une faune riche sous tous les climats: Rhyacophilines, Philopotamides, Psychomyines, Ptilocolépines, Hydroptilides, Hélicopsychines, Beraeines, etc.

Il existe certainement des relations assez complexes et encore peu claires entre la vélocité, le débit, la température et l'oxygénation de l'eau. Mais il se trouve que tous les groupes qui sont sortis du milieu torrentiel froid et ont colonisé des eaux tranquilles ou de très faible vélocité ou débit ou de température assez élevée, montrent généralement de plus grandes spécialisations morphologiques que les groupes qui y sont restés.

Deux lignées seulement semblent s'être adaptées au milieu hypertorrentiel. Les Rhyacophila du groupe de hobsoni et les Himalopsyche vivent en abondance dans les torrents glaciaires himalayens, dont le débit est énorme, la température minimale et la vélocité et l'agitation proprement terrifiantes.

\section{Rythme différenciel de l'évolution chez les larves et chez les adultes}

I a "Chart I") donne un arbre phylétique de l'ordre des Trichoptères, le premier qui soit apparu dans la littérature. Cet arbre reproduit dans ses grandes lignes l'opinion générale des auteurs sur la classification des Trichoptères, mais il est probable que des changements de détails y seront apportées par des études ultérieures. Cet arbre illustre 
en particulier l'opinion de Ross, apparaissant plusieurs fois dans le texte, que les caractères servant de base aux divisions majeures de l'ordre, aux divisions familiales entre autres, sont apparus d'abord chez les larves et ensuite chez les adultes. A la page 20, il est expliqué, avec référence à la "Chart I") que "the adults seem to have changed scarcely at all, while the larvae were evolving casemaking habits at a relaltively rapid rate). En effet, sur la "Chart $x$ ", les premières divisions de l'ordre sont basées sur des caractères larvaires et plus haut seulement apparaissent les caractères imaginaux. Ceci ne serait convainquant que si c'était la seule façon de procéder. Or, je me fais fort d'illustrer un tel arbre à l'aide de caractères imaginaux seulement, ce qui prouverait peut-être que les adultes ont évolué rapidement, alors que les larves restaient statiques. Voici un premier exemple, et nous en trouveront d'autres par la suite où la nature de faits donnés comme établis n'est que l'expression de la façon de procéder du chercheur.

L'idée que les larves ont évolué plus précocement et donc plus rapidement que les imagos est certainement intéressante et concerne la génétique aussi bien que la systématique. Génétiquement, la question se pose de la façon suivante: des mutations, nombreuses et importantes, peuvent-elles modifier les stades jeunes, tout en laissant les adultes intacts? Etant systématicien, je ne me risquerai pas à donner une réponse directe à cette question. Ross y répond par l'affirmative, inais de façon indirecte aussi. Les larves ont été soumises dès le début t̀ une forte pression de sélection, qui a été une des causes de leur différenciation précoce, alors que "the adults were static because they remained virtually in the same environment during the entire evolutionary period. That environment would be the shaded banks of cool, woodland streams, where today we find the adults of primitive families disporting themselves in very much the same manner as they probably did eons agon.

Mon objection de systématicien est que, dans l'ordre des T'richoptères, de même que dans la classe des Insectes toute entière, les adultes sont généralement beaucoup plus différenciés entre eux que le sont les larves - qui sont plus conservatrices - et cela aux niveaux familiaux, génériques, aussi bien que spécifiques. Or, comment expliquer cela, la pression de sélection ne s'étant jamais exercée sur eux de façon sensible, puisqu'on les trouve aujourd-hui dans les mêmes conditions où vivaient leurs plus lointains ancêtres?

Probablement faudrait-il admettre que la pression de sélection se manifeste sur tous les stades, quoique de façon peut-être moins facile à déceler sur les adultes que sur les stades jeunes (les imagos, toutefois, sont très sensibles à la température de l'air) et que ceux-1à, par surcroit se trouvent aussi influencés par des mutations ne semblant modifier que les larves. Ceci impliquerait que les adultes se sont différenciés en même temps que les larves, mais plus rapidement que ces dernières et que les caractères imaginaux sont, à tous les niveaux, aussi fondamentaux que les caractères larvaires.

\section{Reconstitution des types morphologiques ancestraux}

La méthode proposée par Ross est au fond la même que celle des (photo-robots" employées par la police pour l'identification des cri- 
minels. Il s'agit de sélectionner un à un les caractères primitifs fournis par les membres d'un certain groupe et dispersés dans le cadre de ce đernier et de les réunir en un schéma, (an imaginary creation of our reasonning", dont on aura tout lieu de penser qu'il se rapproche quelque peu de l'ancêtre du groupe en question.

Cette façon de faire est réellement excellente, mais, très malheureusement, Ross ne la met pas lui-même en pratique dans son oeuvre. Dans ses considérations phylétiques, l'auteur admet constamment, et sans donner de justification, que les espèces aux génitalia les plus simples, sont, par définition, les plus primitives. Une des conséquences en est qu'il n'a jamais établi d'homologies entre la plupart des appendices communs aux trois familles étudiées.

\section{La simplicité, critère de primitivisme.}

Cette façon de procéder me parait réunir plusieurs erreurs. I) Tout d'abord, je ne pense pas que 1'on puisse se baser sur un critère, sur une méthode passe-partout, pour établir une hiérarchie des degrés de spécialisation. Les voies qu'a emprumpté la Vie pour se diversifier sont décidément trop tortueuses. 2) La notion de simplicité et son antithèse, celle de complication, confondent deux choses bien différentes, qui n'ont pas été séparées par Ross: une armature génitale peut être simple ou complexe par le nombre, grand ou petit, des appendices présents, mais elle peut l'être aussi par la forme, simple ou complexe, des mêmes appendices. 3) Malheureusement, il semble bien que les choses se soient précisément passées dans l'ordre inverse de celui établi par Ross: la simplicité des génitalia parait être généralement 1'indice d'un assez haut niveau de spécialisation.

A considérer dans chaque famille les espèces que l'auteur considère comme les plus primitives, on ne saurait se garder de l'impression de quelque chose qui sonne faux. Dans le cas des Rhyacophila de la branche I, par exemple, les espèces, stigmatica et lobifera entre autres, ont développé des formes si particulières, si inorthodoxes dans le cadre du genre, que l'on ne peut les considérer comme les descendants d'un rameau primitif. Dans le cas de Protoglossa et Sinoglossa, la simplicité des génitalia est si grande qu'elle ressemble à un véritable dépouillement et ne peut s'expliquer logiquement que par la réduction.

\section{Le manque de recherche des homologies}

A la page 9, l'auteur donne une description d'une armature génitale qu'il considère comme primitive dans le cadre de l'ordre tout entier. Ce schéma est certainement exact, mais il est beaucoup trop incomplet pour être utile et il est impossible d'admettre que la présence, au Xme segment (au. XIme selon NrELSEN) d'appendices autres que les appendices praeanaux (cerci) soit le résultat d'acquisition indépendantes des différentes lignées. D'autre part, rien n'est dévoilé au sujet de la constitution de l'appareil pénial.

A comparer certaines des structures des trois familles étudiées, on se demande, par exemple, pourquoi les lobes latéraux du Xme segment de Chimarra ne seraient pas les homologues des branches latérales de Kisaura et de Philopotamus. Pourquoi les tendons dorsanx latéraux 
et la "ventral keel) de Eomystra ne seraient pas des modifications des tendons dorsaux et ventraux d'autres Glossosoma. Pourquoi les "dorsal flaps) de Eomystra ne seraient pas les paramères, présents chez tant de familles de 'Trichoptères?

Dans ma revision des Limnophilides, j'ai montré que l'évolution des'génitalia chez cette famille semble se faire selon. la ligne suivante: à partir de génitalia complexes par le grand nombre des appendices dont la forme est simple, on assiste à une simplification par perte ou fusion de certaines pièces, puis à une recomplication secondaire, je dirais presque à un rajeunissement de l'armature, par complication de la forme des appendices restants. Or, ce fait est très génćral en biologie. Un des premiers chercheurs qui en ait pris conscience, WILLISTON, l'a même formulé dans les termes généraux de la "loi» qui porte son nom: "The parts in an organism tend toward reduction in number, with the fewer parts greatly specialised in fonction". C'est selon cette ligne, à mon avis, qu'il conviendrait de reconstituer l'évolution des groupes étudiés, en tenant compte, bien entendu, des exceptions tonjours possibles - chez les Hydrobiosines peut-être - c'est-à-dire en se gardant de l'élever elle même au rang de critère.

Pour en revenir aux considération phylétiques de Ross, je dirai craindre fort que beaucoup d'entre elles ne soient entièrement faussées et cela dès la base, par ces deux erreurs, le manque de recherche des homologies et 1'identification de la simplicité avec la primitivisme. Néanmoins, certaines séquences morphologiques, celles mises en valeur chez Wormaldia et Glossosoma s. str., par exemple, sonnent juste à la lecture, car nous assistons ici à une complication progressive de formes qui sont primitivement simples dans le cadre de ces genres.

\section{Construction des arbres phylétiques}

L'ouvrage de Ross présente un grand nombre d'arbres phylétiques qui non seulement illustrent les séquences morphologiques expliquées dans le texte, mais constituent aussi la base indispensable aux considérations zoogéographiques.

Or, à suivre en détail la composition de certains de ces arbres phylétiques et les caractères sur lesquels sont basées les divisions successives, se dégage là aussi une impression de malaise et d'artificialité. D'autre part, beaucoup d'arbres ont l'aspect de peignes, c'est-à-dire sont formés d'une lignée centrale rectiligne dont se détachent, à intervales réguliers, des espèces isolées ou de petits groupes compacts. Il est évidemment possible que l'évolution des unités systématiques étudiées ait eu lieu de cette façon "pectiniforme), mais la fréquence de ce fait augmente l'impression d'artificialité mentionnée plus haut et dont je me suis appliqué à rechercher la cause.

A considérer un exemple typique, l'arbre phylétique des Rhyacophila du groupe de sibirica par exemple (Chart $2 \mathrm{r}$ ), il apparait que les divisions sont basées sur des caractères de faible importance, souvent sur des détails, qui sont toujours considérés 1'un après l'autre. On se demande donc pour quelle raison tel détail a été choisi plutôt que têl 
autre dans le vaste tableau des caractères offerts par le groupe. Et il ne semble bien qu'il ne l'a été que parce qu'il permet des divisions commodes. Et la succession de ces détails, pris un à un et placés bout à bout, forme un ensemble linéaire et artificiel, car certains caractères apparaissant en fin de lignée, comme les ulateral flanges of the phalicata), sont déjà présents, mais non cités, à la base de la lignée. I1 semblerait donc que si la lignée centrale du groupe de sibirica apparait linéaire c'est simplement parce qu'elle a été construite de façon linéaire et que l'auteur a projeté sa façon de penser dans sa construction.

Par jeu et par curiosité, je me suis aussi appliqué à dessiner un arbre phylétique du groupe de sibirica, en utilisant uniquement les figures de Ross afin de voir si une autre solution était possible. Or, il m'est apparu que, dès la base, le groupe est divisible en deux lignées basées sur trois caractères fondamentaux pour chacune d'elle, lignées divisibles et redivisibles plusieurs fois sur la base de caractères qui sont de moins en moins importants lorsqu'on s'approche de l'extrémité des rameaux. Sans doute, ai-je aussi projeté ma façon de penser dans ma construction, ce dont je suis personnellement mauvais juge, mais je trouve que mon arbre phylétique forme un ensemble plus cohérent, plus logique jusque dans les détails et surtout moins extraordinaire que celui de Ross.

Un autre exemple de construction que je juge boiteuse est celui des premières division des Glossosomatines (Chart 27). Ross constate que les Anagapetini ont un mésépisternum montrant une suture. Cela le sépare d'emblée du tronc de l'arbre. Les trois autres groupes, Glossosomatini, Agapetini et Protoptilines possèdent chacun des caractères que n'ont pas les deux autres. Le contraire serait étonnant, mais cela prouverait néanmoins qu'aucun d'eux n'a donné naissance à un autre, mais que tous les trois se sont différenciés indépendamment à partir d'un ancêtre commun. Ceci est vraisemblable à la lecture, mais se trouve contredit de façon flagrante par la considération d'autres caractères non cités dans l'arbre et dont Ross ne se préoccupe pas.

Les Anagapetini semblent posséder des tendons dorsaux et ventraux à l'appareil pénial et peut-être aussi des "tendons flaps" qui, selon Ross seraient des développement finaux de la lignée des Anseriglossa. Los 1 gapetini présentent des génitalia si différents de ceux des Glossosomatini que l'on est forcé d'admettre que le clivage entre ces deux groupes a dû être très précoce dans l'histoire de la famille. Alors que les Protoptilines ont des génitalia si voisins de ceux des Glossosomatini, qu'on ne peut les faire dériver d'un ancêtre commun avec Glossosoma, mais d'un ancêtre qui était déjà un Glossosoma.

Ein somme, il me parait que les arbres phylétiques de Ross sont en fait des représentations graphiques de tables dichotomiques, ignorées conme telles de leur auteur; ils sont composés de la même manière et dans le mêtrie esprit, c'est-à-dire se basent sur des caractères de détail, faciles, précis, sautant à l'oeil, mais le plus souvent superficiels et par conséquent artificiels. Or, la reconstruction de la phylogénie d'un groupe doit être basée sur des caractères aussi fondamentaux que possible, caractères qui sont le plus souvent les plus difficiles à déceler et même à décrire. Et, à mon expérience, l'interprétation des caractères fondamentaux abouti à des constructions phylétiques n'ayant pas la facilité de celles qu'a présentées Ross. 


\section{Systématique}

\section{Philopotamidae}

Cette famille montre une grande homogénéité dans ses caractères, ce qui rend difficile une reconstitution de sa phylogénie. Ross classe dans une sous-famille spéciale Paulianodes, dont le IXme segment est annulaire et distinct du Xme et tous les autres genres dans une deuxième sous-famille, les Philopotamines, dont les membres montrent les IXme et Xme segments soudés et intégrés 1'un dans l'autre. Les Philopotamides présentent des caractères tels que 1'auteur les fait tous dériver d'un ancêtre commun et cosmopolite, le "Sortosa ancestorn, dont la descendance aurait produit, par isolement géographique, les genres actuellement connus, qui, pour la plupart, sont restés dans leur habitat ancestrai.

A ceci, j'ajouterai que, par son IXme segment annulaire, Paulianodes est le genre le plus primitif, non seulement de la famille qui nous occupe ici, mais peut-être du sous-ordre des Annulipalpes tout entier. Ross a donc en raison de l'isoler dans une sous-famille spéciale. Mais, j'élèverai deux objections à cette classification :

I) I1 est visible que la fusion des deux derniers segments ne s'est pas produite de la même manière chez Chimarra et chez les autres Philopotamines. Chez Chimarra, le Xme segment est soude au IXme, mais en géneral pas imbriqué dans ce dernier, donit le bord dorsal apical est souvent rectiligne, alors que le bord antérieur est profondément échancré. Chez les autres philopotamines, le bord antérieur du IXme segment n'est pas échancré et le Xme segment est non seulement soudé au IXme, mais fortement imbriqué dans ce dernier, dont la partie dorsale a presque disparu, mais à partir de l'arrière senlement. Cola prouve, à mon avis, que Chimarra et son proche parent Protarra, ne sont pas issus du "Sortosa ancestor", mais sont apparus independamment de ce dernier. Chimarra et Protarra devraient être considérés comme des Paulianodinae qui devraient alors s'appeler Chimarrinae Milne.

2) Ross déclare que les branches latérailes du Xme segment se sont développées indépendamment chez Philopotamus et Tylakion d'une part et chez Kisaura d'autre part, car elles ne sont pas insérées au même point du Xme segment. Or, Protarra montre aussi des branches au Xme segment, que l'auteur dessine mais dont il ne parle pas. Chimarra possède des lobes latéraux au même segment, que Ross dessine et dont il parle, mais sans poser ia question de leur origine. D'autre part, il s'est révélé, après la parution ide l'ouvrage de Ross, que Sortosa s. str. possède aussi des branches au Xme segment. I1 est donc regrettable que le critère de la simplicité ait empêché l'auteur américain d'homologuer les branches du Xme segment des differents genres, car il est impossible d'admettre que sur I3 genres actuellement connus, six aient développé indépendamment des organes semblables.

Du même coup, me semblent remises en question la composition de la descendance du Sortosa — qui doit s'appeler Dolophilodes - ancestor aussi bien que 1'âge et l'origine néotropicale de Chimarra. 


\section{Rhyacophila}

Une reconstitution de la phylogénie de Rhyacophila est certainement une entreprise très ardue, car non seulement, ce genre abrite environ 250 espèces connues, mais montre des variations de nature particulière. Certaines structures sont étonnamment stables et forment un cadre très rigide à des variations d'importance moindre, qui elles, présentent une amplitude et une fantaisie considérables. Ce contraste est parfois presque paradoxal et pose un intéressant problème des deux points de vue systématique et génétique.

Dans son essai de reconstitution, Ross a fait montre de beaucoup d'originalité en basant sa classification, non pas sur la forme des appendices, mais sur un ensemble de caractères que les auteurs avaient négligé avant lui, sur les connections du Xme segment et de l'appareil pénial. A quelques réserves près, les résultats sont excellents et ont permis à 1'auteur de s'avancer très loin dans la classification des divisions fondamentales de ce grand genre.

Les réserves que je ferai sont les suivantes:

I) I'auteur n'a pas eu une claire conscience des caractères primitifs, admettant que les espèces les plus primitives sont fondamentalement les plus simples. Or, la bande tergale et la bande apicale sont certainement des caractères primitifs et non des acquisitions faites en cours d'évolution dans le cadre du genre.

2) La branche $x$, basée sur l'absence des deux caractères qui viennent d'ĉtre mentionnés, est certainement polyphylétique, ce qui est clairement indiqué par son hétérogénéité.

3) I a branche 9 , où la bande apicale s'articule avec la sclérite anal, est certainement aussi polyphylétique, comme l'indique également son hétérogénéité. A examiner les figures, il apparait que ce caractère est important chez verrula et vagrita, mais on se demande quel rôle mécanique il peut bien jouer chez angelita, willametta et retracta par exemple ct, par conséquent, quelle est sa valeur phylétique. D'autre part, chez plusieurs espèces que Ross ne place pas dans la branche 6 , chez certaines formes du groupe de coloradensis, la bande apicale s'articule largement avec le sclérite anal!

4) L'auteur a accordé une valeur absolue aux caractères sur lesquels il s'est basé, alors qu'il aurait eu intérêt en à garder une vue plus souple et à considérer aussi la forme des appendices, qui demeure parfois assez constante dans des groupes peu homogènes par d'autres caractères.

Sur ces bases, certaines améliorations peuvent être apportée à la classification de Rhyacophila: I) L'ordre des branches serait à modifier la branche 4 étant probablement la plus primitive. 2) Beaucoup des groupes et espèces isolées peuvent être insérées dans les branches. Ainsi, les groupes de anatina et de vagrita et peut-être aussi viquaea pourraient prendre place dans la branche 4 ; torva se trouve être.l'espèce la plus primitive du groupe de nigrocephala et celle - dont l'absence est déplorée par Ross - qui permet de voir que le "sagittal process) est un (basal process). modifié, ce dont on pourrait d'ailleurs se douter en voyant remingtoni; ecosa est sans doute voisin de stigmatica; les groupes de vobara et willametta se classent dans la branche $2 ;$ celui de angelita dans le groupe de sibirica et alexanderi au voisinage de betteni. 
Remanié de cette façon, le genre se réduit à sept branches, peutêtre réunissables en trois trones qu'il me parait impossible de rattacher entre eux. Demeure malheureusement un résidu de groupes et d'espèces isolées et inclassables, lobifera, alberta, fletcheri, truncata, etc., et les groupes de nigrocephala et de stigmatica s. 1. qui pourraient peut-être être élevés au rang de branches aussi.

\section{Glossosoma}

Tel qu'il a été redéfini par Ross, le genre Glossosoma se compose d'un certain nombre de sous-genres montrant des caractères divers, mais assez visiblement dérivés les uns de autres pour que l'on puisse espérer reconstituer la différenciation du genre de façon harmonientse.

Or, c'est chez Glossosoma que l'identification de la simplicité au primitivisme, de même que l'absence de recherches dans les homologies ont eu les effets les plus regrettables. D'autre part, l'auteur ne semble pas avoir mis à l'étude de ce genre le même soin qu'il a réservé aux autres. Les négligences et les contradictions ne sont pas rares. Par exemple, dans la lignée de Anseriglossa, à deux reprises, les figtrres destinées à illustrer une séquence morphologique expliquée dans le texte, la contre-disent précisément.

Je me bornerai ici à faire une comparaison entre la classification de Glossosoma s. str. et de Eomystra. Dans le second de ces sous-genres, Ross classe les espèces par la constitution de l'appareil pénial. Il montre comment des prolongements issus de la base de cet appareil, les (dorsal flaps), s'individualisent progressivement, en même temps qu'apparaissent des tendons dorsaux lateraux qui s'ćpaississent puis se soudent progressivement à cette même base. Le Xme segment, de forme complexe qu'il est chez les formes primitives, se simplifie considérabilement. Chez Glossosoma, l'appareil pénial ne montrant pas de variations intéressantes, l'auteur classe les espèces par la forme du Xme segment, qui de simple qu'il est chez les espèces primitives, se complique progressivement pour devenir asymétrique en fin de lignéc.

Un doute se fait jour lorsque l'on considère les variations de la forme du Xme segment se faire en sens inverse chez les deux sousgenres. Lies espèces n'auraient-elles pas été disposées en sens inverse parce que l'auteur a basé ses deux classifications sur deux appareils différents? Et ce doute se ferait certitude si l'on considérait que les "dorsal flaps) de Eomystra sont les paramères qui se soudent progressivement à la phallobase, alors que s'amincissent les tendons dorsaux pour donner plus de liberté de mouvement à l'appareil pénial. L'ordre dans lequel sont classés les Eomystra se trouverait inversé et les variations de la forme du Xme segment se ferait parallèlement chez Glossosoma et Eomystra et conformément à ce que j'explique à la page 102.

A mon avis, dans l'état actuel de nos connaissances, il cst aussi difficile de classer les sous-genres de Glossosoma de façon satisfaisante que d'arranger les branches de Rhyacophila; trop d'intermédiaires nous manquent pour expliquer le passage d'une structure à l'autre. Un des points les plus importants serait de démontrer si les "tendons flaps" des Glossosoma s. str. sont, ou non, les homologues des pièces appelées "cerci" chez Ripaeglossa et "apical band" chez Rhyacophila. 


\section{Zoogéographie}

Les trois derniers chapitres, qui sont certainement parmis les plus brillants de l'ouvrage de Ross, sont consacrés à la zoogéographie des trois familles étudiées, de même qu'à un essai de reconstitution chronologique des dispersions.

Commene, d'une part, il est plus difficile - car trop facile - de critiquer les arguments zoogéographiques que les séquences morphologiques et comme, d'autre part, je n'ai pas encore épuisé la richesse des textes en question, je me bornerai ici à quelques critiques et commentaires sur certains points particuliers. Dans les derniers paragraphes, je me suis laissé entrainer hors du cadre de cette critique, mais j'espère que mes commentaires, qui ont été du reste inspirés par les considérations de Ross, présenteront quelque valeur constructive.

\section{Systématique et zoogéographie}

Pour Ross, Chimarra moselyi, les Anagapetini, Glossosoma taeniatume et sellatum, par exemple, sont des formes relativement très prinitives, des fossiles vivants. Pour moi, ce sont des espèces très spécialisees! Mais, considérons plutôt, une fois encore, les Rhyacophila du groupe de sibirica. Sur la "Chart 2I", le groupe révèle s'être diversifié de façon pectiforme, à partir d'une lignée principale unique et rectiligne. Sur la "Chart 4I", il apparait que les espèces sont réparties en proportions approximativement égalies des del1x côtés de l'ancien isthme de Behring. Pour suivre les développements de la lignée principale, nous sommes conduit à sauter alternativement 'd'un continent à l'autre, en un véritable mouvement pendulaire, qui, pour le groupe, aurait duré une bonne partie du Tertiaire. Et cela, une fois encore, sonne faux. Sur le diagramme que j'ai moi-même dessiné, sur d'autres bases morphologiques mais à l'aide 'des figures de Ross, j'obtiens une lignee asiatique, le sous-groupe de sibirica et une lignée américaine, le sous-groupe de unimaculata, qui ne semblent nullement souffrir de déracinement chronique, mais possèdent tous deux plusieurs branches ayant émigré sur le continent voisin, à trois périodes différentes, mais à une setule reprise chacune. Cela ressemble peu a la reconstitution de Ross.

Il importerait donc avant toute chose, que les auteurs se mettent d'accord sur la phylogénie des entités taxonomiques qu'ils étudient. Il faudrait que nous ayions une vision claire et unifiée de ce qu'ont pu être les caractères primitifs du progéniteur théorique de l'ordre, de même que des ancêtres schématiques des diverses familles. Il importerait également que nous connaissions les différentes directions d'évolution dans lesquelles se sont engagées les diverses lignées et dans quel sens se font en général les modifications graduelles des caractères. Tant que les abords théoriques de ces divers probièmes n'auront pas eté débroussaillés, les reconstitutions zoogéographiques ne prođuiront que confusion.

\section{Les ponts de Behring}

A la page rI2, Ross remarque que l'Asie possède deux faunes, l'une liabitant au nord des grands déserts centraux et l'autre au sud 
de ces derniers. Mais il affirme son intention de traiter l'Asie comme une unité faunistique, à cause du recouvrement de ces deux faunes au Japon. I1 est, à mon sens, regrettable que l'auteur n'ait pas tenu compte de ce caractère bipartit de la faune asiatique. La valeur de ses considérations sur les ponts de Behring en aurait été singulièrement augmentée, car les rapports mutuels des trois faunes, asiatiques et néarctique, sont de nature différente et la comparaison de ces rapports est pleine d'enseignements.

Entre les faunes orientale et paléarctique il y a eu des dispersions à des périodes très reculées, qui ne peuvent se placer que dans le Mésozoïque, mais des échanges d'espèces le long du bord du plateau tibétain, au Péistocène 'certainement. Il y a "overlapping" des deux faunes, non seulement au Japon, mais en Chine et en Sibérie orientale.

Entre les faunes paléarctique et néarctique, il $\mathrm{y}$ a eu des passages d'espèces, à plusieurs reprises, dans les deux sens et à des époques très diverses.

Mais plus intéressants sont les rapports entre les faunes orientale et néarctique. Il ne semble pas avoir eu de dispersions de formes néarctiques dans la région orientale, alors que des espèces orientales ont pa'ssé dans la zone néarctique, mais en très petit nombre et dans le sens Ouest-Esst seulement. Sur 24 lignées typiquement orientales, contenant ensemble plus d'une centaine d'espèces, sept seulement ont des représéntants en Amérique du Nord et ces dernier's sont au nombre de douze.

Pourquoi si peu de lignées orientales et tant de groupes paléarctiques et néarctiques ont-ils traversé le pont de Behring. La raison en est assez obvie. Un petit nombre seulement de formes orientales, les plus septentrionales seulement, vivaient à proximité de l'isthme, alors que les formes paléarctiques et néarctiques, $y$ ayant un accès géographique beaucoup plus large, l'ont traversé en plus grand nombre. Si aucune espèce néarctique n'a passé dans la zone orientale, c'est pour la même raison.

Pourquoi aucune lignée orientale, ayant passé dans la région néarctique n'est-elle retournée en Asie? La raison, ici aussi, apparait d'ellemême. Huit espèces seulement habitent l'Ouest américain et, sans doute, ce nombre est-il statistiquement trop faible pour avoir offert de sérieuses chances de redispersion.

Elargissant le problème, j'ai comparés entre eux tous les arbres phylétiques et les essais de reconstitutions zoogéographiques de Ross Après avoir éliminés Chimarra et les Agapetini, dont la classification me parait douteuse et que je ne puis remplacer par une autre et après avoir remanié les "Charts" 40, $4 \mathrm{I}$ et 43 , sur la base d'autres arguments phylétiques, je constate que toutés les lignées d'âge Crétacé tardif et Paléocène ont un comportement assez semblable à celui des groupes orientaux. Ces lignées ont soit tous leurs effectifs, soit la majorité de ceux-ci dans une seule zone faunistique. Certaines d'entre elles ont vu un petit nombre de leurs branches passer, à des périodes ultérieures, dans une autre région, mais ces branches s'y sont assez peu différenciées et n'ont vu aucun de leurs descendants retourner dans la zone ancestrale. Airisi, aucun rameau secondairement établi dans l'est américain n'est retourné dans les Montagnes Rocheuses, aucune branche néarctique d'origine asiatique n'est revenue en Sibéric et aucune lignée néarctique établie en Asie n'est rentrée en Amérique. 
Ces faits ne présentent que deux exceptions, que nous examineront plus loin. J'ajouterai que le comportement de ces nombreuses lignées. forme un ensemble si homogène et si cohérent, au contraire de ce qu'affirme Ross, que cette régularité m'amène à douter un peu de la correction de mes résultats.

La raison de cet état de chose est le même que dans la cas des lignées orientales. Il semblerait qu'aucun rameau qui s'est établi après le Paléocène en région étrangère ne s'y est différencié suffisamment, n'a donné naissance à un nombre d'espèce assez élevé, pour que, selon les lois de la probabilité, quelques-uns de ses descendants ait eu la chance de se retrouver dans des conditions géographiques et écologiques favorables à un retour dans la zone ancestrale.

Pourquoi ces rameaux ont-ils si peu proliféré après leur dispersion? Cela peut s'expliquer par un rythme d'évolution assez lent, donc par le manque de temps, ou, plus probablement, par la compétition écologique avec d'autres groupes antérieurement et plus solidement établis dans la région.

Les seules exceptions sont les Eomystra dulkejti et intermedium, dont les lignées ont connu deux dispersions post-paléocènes. Ces formess habitent les plaines froides plutôt que les montagnes et leur redispersion s'explique par les glaciations pléistocènes. Les Rhyacophila du groupe de acropedes et Glossosoma s. str. constituent aussi une exception partielle; leur cas sera examiné dans le paragraphe suivant.

Mais, quelle que soit la valeur de ces inférences, il apparait bien que le va et vient par le pont de Behring durant le Cénozoïque est une impression d'ensemble, mais qu'il n'a pas eu lieu à l'intérieur des groupes, car chaque lignée, prise individuellement, ne l'a traversée qu'une seule fois (sauf les deux Eomystra). Je doute fort que, chez les 'Irichoptères monticoles, on puisse reconnaitre sept dispersions cénozoïques. J'en estimerais le nombre à trois et maximum à quatre: palécène, oligocène-miocène et pléistocène peut-être bien et aussi pliocène, possiblement.

Age des zones faunistiques actuelles et des lignées qui en déterminent. l'existence ${ }^{1}$.

L'hémisphère septentrional apparait composé de quatre zones faunistiques: l'Asie paléarctique, l'Asie orientale, l'Europe et l'Amérique néarctique.

Grâce aux études de Ross, nous connaissons maintenant l'existence de lignées d'âge mésozoïque tardif et paléocène; peu nombreuses et dont je retiendrai le "Dolophilodes ancestorn; Wormaldia, les ancêtres des branches $2,3,4,6$; et 7 et du groupe de stigmatica du genre Rhyacophila, les ancêtres des tribus glossosomatides et les progéniteurs. des sous-genres de Glossosoma et de Synagapetus et Agapetus au sens. classique.

Il se révèle que ces lignees ont connu de vastes dispersions crétacées et paléocènes et que chacune a peuplé plusieurs des zones faunistiques mentionnées plus haut de lignées-filles 'qui, comme nous l'avons.

I. Dans ce paregsaphe, j'ai adopté la chropologie générale proposée par Ross, qui parait vraiscmblable, quoiqu'ell a ait une base bien étroite: la composition des faunes orientale et australienne datées par 1'âge des ares mélanésiens. 
vu au paragraphe précédent, sont, soit strictement localisées dans une seule de ces zones, soit ont le gros de leurs effectifs dans une regrion et de menus rameaux dans une ou deux autres. Ces lignćes-filles sont nombreuses et je ne pense pas inutile d'en donner une liste, en régligeant les espèces isolées dont l'origine est indéterminable. Mais, à certaines espèces orientales isolées que j'ai retenues, s'ajouteront de nombreuses formes non décrites, qui en feront des groupes ${ }^{2}$.

\section{EUROPE}

Philopotamus

Wormaldia (trois groupes réunis)

* Glossosoma s. str.

Synagapetus gr. de armatus

Agapetus gr. de fuscipes et comatus réunis
Rhyacophila groupes de

tristis

stigmatica s. str.

vulgaris

philopotamoides

glaerosa

\section{ASIE PALÉARCTIQUE}

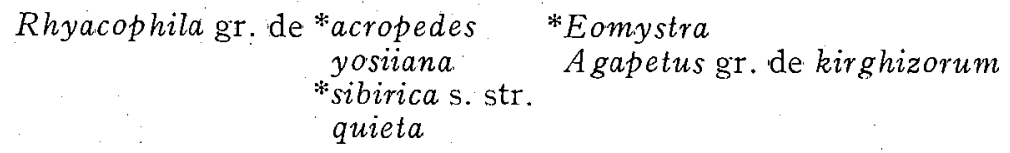

ASIE ORIENTALE

Kisaura

*Dolophilodes

* Wormaldia gr. de moesta

* Doloclanes

Gunungiella

*Himalopsyche

* Apsilochorema

Protoglossa-Sinoglossa

Lipoglossa

Muroglossa

* Glossosoma s. str.

Synagapetus gr. de mitis

* A gapetus gr. de bidens
Rhyacophila gr. de anatina

curvata

divaricata

castanea

scissa

truncata

hobsoni

falita

*lieftincki

naviculata

*nigrocephala

AMÉRIQUE NÉARCTIQUE

Sisko

Fumonta

Wormaldia gr. de anilla

Rhyacophila gr. de nevadensis

$$
\begin{aligned}
& \text { vofixa. } \\
& \text { vobara } \\
& \text { willametta } \\
& \text { fuscula } \\
& \text { rotunda. }
\end{aligned}
$$

Rhyacophila gr. de invaria hyalinata carolina

* unimaculata

Anagapetus

betteni

Agapetus gr. de celatus 
Sur $5^{8}$ lignés mentionnées ici, 44 sont localisées dans une seule région, i2 ont de rares représentants dans une autre zone et deux seulement, Glossosoma s. str. et les Rhyacophila du groupe de acropedes sont réparties en proportions approximativement égales dans deux régions.

Il semble donc probable que les quatre zones faunistiques, telles que nous les connaissons actuellement, ont été déterminées au Crétacé par l'apparition 'des Mignées-filles đu "Dolophilodes ancestorn, des ancêtres des branches de Rhyacophila et des tribus glossosomatides, et que cette individuatisation s'est poursuivie au Paléocène par l'apparition des groupes d'espèces de Wormaldia et de Rhyacophila, des sous-genres de Glossosoma et de Agapetus et Synagapetus. Sur cette base, nous pouvons donc assigner à ces zones faunistiques un âge minimum crétacé. Si elles se sont différenciées à une période anitérieure, les éléments nous manquent totalement pour la idéterminer.

Réciproquement, pouvons-nous inférer que les lignées-filles qui n'ont pas d'ancêtres crétacés ou paléocènes connus, sont de même âge que celles dont les progéniteurs ont été 'da'tés de ces périodes? Cela est, à mon avis, la seule façon de faire, à moins que la phylogénie fournisse une autre réponse de son cru. Ainsi, dans l'oeuvre de Ross, il apparait que l'ancêtre commun des groupes de castanea, hyalinata et philopotamoides et l'ancêtre 3 dans la lignée d'A gapetus seraient oligocène et auraient connus une large dispersion à cette époque. Si ces faits se révèlaient corrects, ce dont je douterais quelque peu, nous pourrions adinettre l'âge oligocène des lignées issues de ces ancêtres.

Certains genres, tels Himalopsyche et Apsilochorema semblent être pré-crétacés, mais leur position phylétique me parait encore trop incertaine pour 'que leurs cas puisse être considérés comme des objections.

Restent à expliquer le cas des Rhyacophila du groupe de acropedes et de Glossosomia s. str. qui sont également répartis dans deux zones. A mon avis, le groupe de acropedes se compose de deux lignées parallèles et d'origine paléarctique qui toutes deux se sont dispersées en Amerique, à une seule reprise. Leur cas est semblable à celui des lignces paléocènes mentionnées plus haut, sauf que le nombre des formes connues habitant la zone ancestrale est aussi faible que celui des espèces connues apparues en zone étrangère après la dispersion. Il y a là un cas particulier, mais sans grande signification. Autre est celui de Glossosoma s. str. qui est florissant en Asie orientale aussi bien qu'en Europe. Les formes primitives sont dans la région orientale et les espèces spécialisées, peut-être issues d'une seule dispersion, vivent en Europe. Dans le cadre de la faune européenne, Glossosoma s. 1. présente des analogies avec Rhyacophila: un des groupes d'espèces, ou sous-genre, est florissant, alors que les autres sont rares et très mal représentés. En Europe donc, Glossosoma s. str. contient une dizaine d'espèces, alors que les autres sous-genres sont représentés par deux Eomystra seulement, d'arrivée plus tardive, pléistocène certainement. Le cas des Glossosoma européens pourrait suggérer que si la plupart des dispersions post-paléocènes entre différentes régions ont connu de maigres succès, c'est à cause de la compétition que les nouveaux arrivés ont trouvé de la part de leurs proches parents morphologiques installés antérieurement et plus solidement qu'eux dans les mêmes biotopes. Cette compétition n'existait pas pour Glossosoma s. str. qui est arrivé en Eirope, bien avant les Eomystra. 
En résumé, les quatre zones faunistiques actuelles sont déterminées par des faunes d'apparition crétacée et paléocène. Depuis cette dernière époque, les échanges faunistiques entre les zones ont été faibles et ont mis en cause des lignées d'importance mineure seulement, sauf les deux cas, peut-être douteux, qui viennent d'être cités. Par contre, des groupes assez importants semblent avoir pris naissance à partir d'ancêtres résidant dans la même zone faunistique, tels les Rhyacophila des groupes de naviculata, liefitincki et falita, qui se sont probablement différenciés à partir d'ancêtres communs dans la région orientale.

\section{Détermination du centre d'apparition dés lignées}

Ce problème est évidemment des plus importants pour les études zoogéographiques, mais il apparait très difficile à résoudre et les résultats obtenus sont souvent incertains. Ross consacre une page de grande valeur à 1'examen des facteurs qui peuvent nous permettre la détermination de ces centres d'apparition. I1 apparait que cet auteur rejette les hypothèses fondées sur des considérations statistiques, mais inclinerait à se baser sur une intégration des données phylétiques et zoogéographiques. Il semblerait, ici aussi, que Ross n'ait pas toujours été fidèle à sa propre méthode, mais ait eu tendance à assigner comme point d'apparition à une lignée, la région où vivent les espèces les plus primitives de cette lignée.

A mon avis, la meilleure façon de faire serait de considérer en effet, les résultats de 1'analyse phylétique et zoogéographique, mais de tenir compte aussi des données statistiques et de reconnaitre une valeur différencielle à ces facteurs, selon 1'âge des lignées considerécs. Cette façon de procéder me parait du reste s'imposer par la nature des faits à expliquer.

Le groupe de nigrocephala du genre Rhyacophila contient r4 espèces orientales et une quinzième, torva, la plus primitive de toutes, vivant en Amérique. Quelle est la région d'origine du groupe? $\mathrm{Ce}$ dernier est-il apparu en Amérique où subsiste un des descendants directs de l'ancêtre et une lignée a-t-elle passé en Asie où elle s'est spécialisée et différenciée? Ou bien, le groupe est-il oriental et c'est par hasard, ou pour des raisons qui nous échappent, que la lignée ayant passé en Amérique se trouve être la plus primitive? I, problème the parait assez facile à résoudre. Lie groupe est certainement oriental et une de ses branches s'est ensuite dispersée en Amérique. Car, s'il était néarctique et arvait ensuite passé en Asie, la branche asiatique serait paléarctique et non orientale, car, comme nous l'avons vu, la région orientale était beaucoup moins ouverte sur le pont de Behring que ne 1'a été la zone paléarctique. Dans le cas des Wormaldia du groupe de moesta, qui est semblable à celui qui vient d'être considéré, Ross penche pour une origine néarctique, car l'espèce la plus primitive de la lignée, gabriella, est américaine. Cette lignée aurait donné naissance à un (widespread holarctic ancestor" dont la descendance aurait subsisté dans la zone orientale, mais se serait éteinte dans la région paléarctique. Cette hypothèse est vraisemblable, mais elle a le defaut d'introduire une entité supplémentaire - une lignée palćarctique dont nous ignorons tout. Pour avoir quelque valeur, une hypothèse ne doit pas être trop flottante mais serrer les faits d'aussi près que possible. Selon moi, le groupe de moesta est également d'origine orientale. 
Pour en revenir au problème de la détermination du centre d'apparition des lignées, je suis d'avis que dans le cas des groupes d'âge post-crétacé supérieur, les facteurs statistiques doivent jouer le premicr rôle. Ainsi, toutes les lignées habitant une seule zone faunistique sont apparues dans cette zone; les lignées ayant le gros de leurs effectifs dans une région y ont pris naissance aussi, quel que soit l'âge de leurs rameaux ayant passé dans d'autres régions; ainsi tous les groupes orientaux et néarctiques sont d'origine orientale. Pour les lignées également réparties dans deux régions, les facteurs phylétiques semblent reprendre le 'dessus. Ainsi, Glossosoma s. str. et les Rhyacophila du groupe de acropedes, dont nous avons déjà parlé, répartis en proportions égales sur deux continents, semblent avoir leur origine où se trouvent leurs formes les plus primitives. Cette façon de procéder conduit certainement à des résultats parfois inexacts, mais c'est celle qui me parait introduire le moins de risques d'erreurs.

Én ce qui concerne les branches d'origine pré-crétacé supérieur, la situation apparait différente et considérablement plus floue. Les zones faunistiques paraissent avoir été autres que celles d'aujourd-hui, les facteurs nécessaires aux inférences sont rares et les considérations statistiques, en particulier, ne sont plus possibles. Une comparaison de l'arbre phyletique ides Trichoptères avec d'autres, retraçant l'histoire de groupes dont les fossiles sont relativement bien connus, les Vertébrés par exemple, suggère l'idée que les Trichoptères primordiaux pourraient avoir connu une radiation adaptative mésozoïque et qu'au Crétacé et au Paléocène, un petit nombre de lignées auraient connu une seconde radiation et donné naissance à une faune nouvelle ayant remplacé l'ancienne et se trouvant être encore actuelle. Les branches phylétiques d'âge mésozoïque qui ont servécu sont beaucoup trop peu nombreuses pour que nous puissions reconstituer, même vaguement, l'histoire et la dispersion le cette faune secondaire hypothétique.

Lorsque, comme le dit Ross, des étapes consécutives d'une séquence morphologique sont visibles dans le même continent, comme dans le cas 'des Sortosa-Protarra-Chimarra, si les déductions de cet auteur étaient exactes, nous pouvons en conclure que le groupe est apparu sur ce continent. Mais, de tels cas sont rares. Chez les Philopotamides, la seule solution qui nous est laissée est l'imagination d'un ancêtre commun et cosmopolite qui aurait produit toutes les lignées qui ont subsisté jusqu'à nos jours. Mais, une telle solution n'est guère satisfaisante, car elle est trop facile et ne nous renseigne pas sur la région où est apparu l'ancêtre en question. Dans le cas des Rhyacophila des branches 2 , 3 et 4 et des ancêtres des tribus glossosomatides le recours à la solution du "widespread holarctic ancestor") est impraticable et rien ne semble pouvoir la remplacer. Il est probable qu'en l'absence de fossiles, bien peu de centres d'apparition mésozoïques nous seront jamais connus.

\section{Effets des glaciations pléistocènes}

Un sujet que Ross a traité très incomplètement est celui des récontes glaciations. Il lui est apparu que ces dernières ont principalement produit des remaniements d'espèces à 1'intérieur des systèmes montagneux, mais très peu de passages d'un système à l'autre. Ceci 
n'est vrai que pour les espèces monticoles de 1'Amérique septentrionale seulement.

A considérer l'immensité des boucliers glaciaires descendus du Nord, il apparait certain que les isothermes ont alors fortement dégringolé vers le sud, en même temps que la faune qui a dû connaitre des déplacements d'une amplitude très grande. Ces glissements ont dût provoquer des télescopages et embouteillages considérables et, sans aucun doute, des faunes entières ont-elles péri dans la mêlée, spécialement celles qui ont rencontré des obstacles géographiques ou écologiques sur leur route. Si ces mouvements de va et vient se sont récllement reproduits quatre fois, il est certain que leurs effets ont éte si complexes qu'il sera impossible de les reconstituer.

Les formes de plaine ont certainement pu se déplacer relativement facilement. Les effets des glaciations sur elles nous apparaissent surtout comme des expansions horizontales, c'est-à-dire parallèles à 1'équateur, mais qui sont en réalité les résultantes de mouvements plus complexes. Il est clair que la mise en place des espèces habitant les grandes plaines septentrionales, principalement: Limnophilides et Phryganéides et la très large répartition de beaucoup des espèces de ces deux familles sont des évènements post-glaciaires.

Les espèces de montagne ont aussi glissé vers le sud, mais leur situation a dû être plus délicate que celle des formes de plaine, les voies de fuite étant moins nombreuses. En Amerique septentrionale, si les effets des glaciations nous apparaissent faibles, c'est probablement parce que ce continent ne possède que deux systèmes montagneux, très largement distants et grosso modo perpendiculaires à l'équateur. Les espèces ont probablement pû glisser relativement librement le long de l'axe des chaines, dans les deux sens. Comme Ross l'indique, les dispersions entre systèmes y sont exceptionelles.

Mais il en est tout autrement en Europe et en Asic. Ces continents possèdent de nombreux systèmes montagneux, souvent isol'és les uns des autres, mais par des distances relativement faibles et se trouvent en général disposés parallèlement à l'équateur. Les mouvements nordsud des espèces monticoles y ont donc provoqué de nombreux passages d'un système à l'autre et beaucoup d'espèces sont restées dans leurs nouveaux habitats ou s'y sont différenciées depuis leur isolement. Ainsi, en Europe, l'Atlas et les Sierras espagnoles, pour ne rien dire des montagnes italiques et balkaniques dont la faune est peu connue, abritent de nombreuses reliques glaciaires, spécialement rhyacophilides et limnophilides. La présence de Acrophylax zerberus et de plusieurs espèces communes ou très voisines des genres Rhyacophila, Drusus, Apatania, etc., dans plusieurs massifs d’Europe centrale est dûe aux glaciations, de même que les si nombreuses répartitions boréoalpines.

En Asie méridionale, la seule partie du continent qui me soit un peu connue, les évènements zoogéographiques pléistocènes sont nombreux et importants: la présence de reliques glaciaires au Bélouchistan pakistanais et à Manipur, la mise en place de la faune du plateaut tibétain, les (injections.) de formes paléarctiques dans la zone orientale, à travers l'axe de 1'Himalaya, Philarctus przewalskii, Limnophilus incisus et kaumarajiva au Pakistan et d'autres espèces non décrites (dont un Asynarchus) dans 1'Himalaya de l'Inde et du Nepal, le glissement de proche en proche le long de 1'Indou-Koush et de 1'Elbourz, parfois 
jusqu'au Caucase, de Rhyacophila obscura, Dolophilodes ornata, de certains Goerodes, Dinarthrum, Apsilochorema, Pseudostenophylax, Stenopsyche et de Stactobia des groupes de martynovi et nielseni.

J'incline même de plus en plus à penser que la présence de nombreuses formes monticoles dans les Western Ghats et à Ceylan, Rhyacophila des groupes de naviculata, curvata et castanea, Doloclanes, Gunungiella, Synagapetus, Stenopsyche, ne sont pas des évènements miocènes comme on l'a affirmé (Ross et HoRA), mais pléistocènes.

Il est donc clair, qu'en Europe et en Asie, les glaciations quaternaires ont été la cause d'une des plus grandes aventures zoogéographiques dont ont été témoins ces continents, ou en tous cas celle qui nous parait dominer leur histoire, à cause de son occurence récente, aucun évènement ultérieur n'étant venu en modifier ou en effacer les effets.

\title{
A comparative study of the genitals segments and their appendages in male Trichoptera
}

\author{
Biol. Skr. Dan. Vid. Selsk. 9, no 5, 1957
}

PAR

\section{ANKER NIEISEN}

I1 est un fait que les pièces génitales du mâle jouent un rôle primordial dans les études taxonomiques sur les Trichoptères, car elles fournissent non seulement la seule base possible pour les distinctions spécifiques, mais aussi des éléments très importants pour les classifications génériques et même familiales. Mais; à parcourir la littérature consacrée aux Trichoptères, on constate que la majorité des descriptions et des figures de génitalia sont insuffisantes pour servir à des études morphologiques et phylétiques et ne sont guère utiles à autre chose qu'à des déterminations de collections.

Pour contribuer à combler cette lacune, NIELSEN à entrepris une vaste étude morphologique et anatomique sur les génitalia du $\sigma^{\gamma}$ d'une trentaine d'espèces danoises appartenant à des familles variées. Le but de cet ouvrage était d'établir des homologies entre les appendices des divers groupes et éventuellement une comparaison avec ceux d'ordres voisins, de même que d'éclairer quelque peu nos connaissances sur la phylogénie de l'ordre et de reconstituer ce 'qu'a pu être l'armature génitale du Trichoptère primordial.

Par ses travaux récents, NiELSEN s'est imposé comme un habile et brillant morphologiste. L'ouvrage commenté ici ne le cède en rien à ses prédécesseurs, ni par la qualité du travail effectué, ni par la somme de connaissances originales qu'il apporte dans un champ de recherches entièrement nouveau. On ne peut manquer d'être émerveillé en imaginant l'habileté et la patience de l'auteur qui est parvenu à comprendre et à interprêter les connections sclérotiques complexes qui réunissent les appendices entre eux, de même que la musculature d'armatures 